\title{
Solving a Novel Multi-Objective Inventory Location Problem by means of a Local Search Algorithm
}

\author{
Carolina LAGOS ${ }^{1}$, Jorge VEGA ${ }^{2}$, Guillermo GUERRERO ${ }^{1}$, Jose-Miguel RUBIO ${ }^{3}$ \\ ${ }^{1}$ Pontificia Universidad Católica de Valparaíso, CHILE, \\ \{carolina.lagos.c; guillermo.guerrero.c\}@mail.pucv.cl \\ ${ }^{2}$ Universidad de Antofagasta, CHILE, \\ jorge.vega@uantofa.cl \\ ${ }^{3}$ Universidad de Playa Ancha, CHILE, \\ jose.rubio.1@upla.cl
}

\begin{abstract}
Problems in operations research are usually modelled as single objective ones even though there exist several goals that should be attained. Multiple reasons of why inherently multi-objective problems are modelled as single objective can be identified: better understanding of the problem features, simplification of the mathematical formulation of the problem, among others. However, summarising (usually conflicting) objectives into one objective function can be a sign of over-simplification in the problem modelling process. In this paper we extend a single objective inventory location problem formulation to a multi-objective one. We consider two conflicting objectives, namely, location cost and inventory cost. As a result, we obtain a complex multi-objective non-linear integer programming problem. Like its single objective formulation, this multi-objective problem cannot be solved by exact methods as the number of decision variables increases. Thus, a simple yet effective multi-objective local search algorithm is implemented in this paper.
\end{abstract}

Keywords: MO Local Search, MO Inventory Location, Combinatorial Optimisation.

\section{Introduction}

Problems in operations research are usually modelled as single objective ones even though there exists, in real world, several goals that should be attained. Multiple reasons of why inherently multi-objective problems are modelled as single objective ones can be outlined: better understanding of the problem features and simplification of the mathematical formulation of the problem, among others. However, summarising (usually conflicting) objectives into one objective function can be a sign of over-simplification in the problem modelling process.

Multi-objective optimisation (MO) aims to optimise two or more conflicting objectives simultaneously. Unlike in single objective optimisation, where solving a problem means to find its optimal solution w.r.t. some objective function, solving a problem in a MO context means to find a set of solutions such that improving an objective without impair at least some other objective is not possible. These solutions are called efficient solutions. Mathematically, a general MO problem can be formulated as follows:

$$
\begin{aligned}
& \min f(x)=\left(f_{1}(x), f_{2}(x), \ldots, f_{p}(x)\right) \\
& \text { s.t. } \quad x \in X \subseteq \mathbb{R}^{n}
\end{aligned}
$$

where $x$ is a vector of $n$ elements and $X$ is the set of feasible solutions. Consequently, $f(x)=y \in Y \subseteq \mathbb{R}^{p}$ is the image of solution $x \in X$ in the objective space.

We say that a solution $x^{1} \in X$ is efficient if there is no $x^{2} \in X$ such that $f_{i}\left(x^{2}\right)<f_{i}\left(x^{1}\right)$ for some $i=1, \ldots, p$. The set of efficient solutions is denoted by $X_{E} \subseteq X$. Consequently, we denote the image of $\hat{x} \in X_{E}$ in objective space as $f(\hat{x})=\hat{y}$, where $\hat{y}$ is called nondominated point. The set of non-dominated points of the MO problem in (1) is $Y_{N} \subseteq Y$.

In this work a model for a MO inventory location model (ILM) problem is proposed. ILM problems aim to integrate strategic decisions with tactical ones. In particular, the ILM considered in this paper seeks to integrate location decision making (strategic) with inventory policies (tactical). We consider two conflicting objectives: On the one hand, we aim to minimize the location/allocation cost, that is, the cost of installing a warehouse and, on the other hand, we want to minimize the inventory cost, that is, the cost of holding products in our warehouses and the cost of processing an order from our customers.

The problem of locating/allocating customer to distribution centres is one of the most studied problems in logistics. Usually, after decision makers determine the locations to be installed, 
the inventory policy is defined. However, this sequential approach is sub-optimal in the sense that the inventory policy is restricted by the network design determined in the previous step. Thus, integrating location/allocation decisions and inventory policies lead to solutions that are more efficient as they consider the entire system as a whole. Figure 1 shows a schema of both the sequential and the integrated approaches.

The remaining of this paper is as follows: In Section 2 we present a brief literature review of previously proposed models for the ILM. Although we include some single objective ILMs, the review is mainly focused on the multi-objective models presented so far. In Section 3 we introduce a novel MO-ILM which is an extension of the single objective ILM presented in [18]. In Section 4, the multiobjective local search we consider to solve the MO-ILM is outlined. In Section 5, the computational experiments performed in this paper are described and the obtained results are discussed. Finally, in Section 5, some conclusions and the future work are outlined.

\section{Inventory-Location Modelling: Literature Review.}

During the last two decade, several single objective ILMs have been proposed in the literature. Different approaches to integrate both strategic and tactical level within the decision making process have been considered $[4,6,7,10,12,15,17,19,20,21,25,26]$. All these approaches comprise all objectives into one single objective function. This has several drawbacks. As mentioned in Section 1, summarizing all the goals into only one objective function can be a sign of oversimplification of the problem modelling process. Moreover, the importance of each

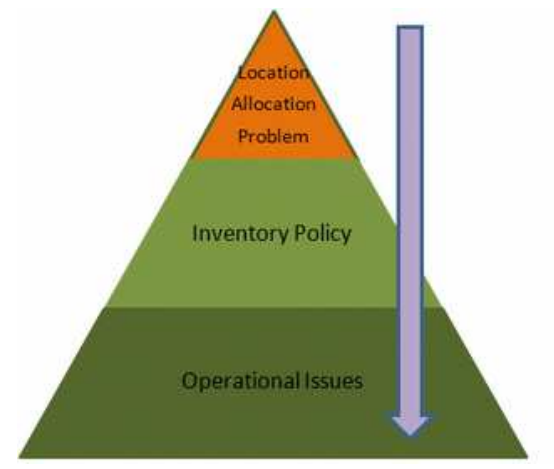

objective can be set differently depending on the decision maker personal preferences. Furthermore, solving the single objective problem will lead us to one solution that is (hopefully) the optimal solution of the problem. However, having only one solution does not give us any insight on the existing trade-offs between the objectives of the problem.

Although less studied, MO-ILMs have also been proposed in the literature (see [1, 3]). In [1] the authors design a distribution network model considering three-echelon and integrating location and inventory decisions. The objectives considered in their model are the overall profit and the level of service as a measure of the customers' dissatisfaction. Authors in [3] propose a MO-ILM considering different transportation modes. Three objectives are considered in their work: total costs, earliness and tardiness, and deterioration rate.

Since in general both continuous and integer decision variables are part of the models above, the ILM problem is usually described as a mixed integer one. Furthermore, objective functions are usually non-linear. Thus, solving this problem to optimality within a reasonable time is simply not possible as the number of decision variables becomes larger. Thus, several authors have considered heuristic methods to approximately solve this problem. For instance, the authors in $[3,5]$ implemented an evolutionary algorithm called NSGA-II. Evolutionary algorithms are also used in [16]

\section{Multi-Objective ILM}

As we mentioned before, the MO-ILM we present in this paper extends from the single objective ILM in [18].

The mathematical model for the MO-ILM that we introduce in this paper is as follows:

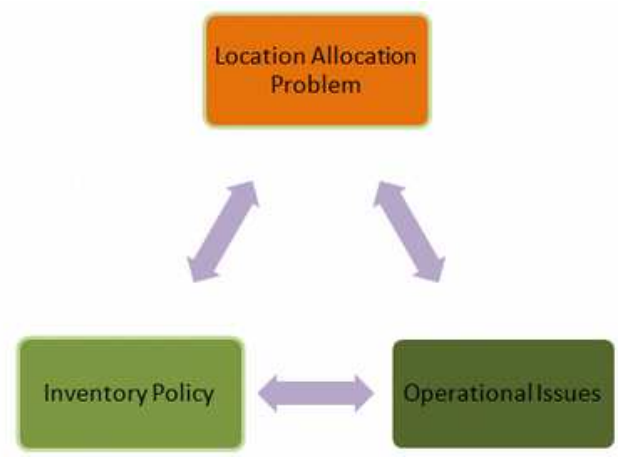

Figure 1. Traditional sequential approach (on the left) versus the integrated approach (on the right). 


$$
\begin{aligned}
& \operatorname{Min} \sum_{i=1}^{N} F_{i} x_{i}+\sum_{i=1}^{N} \sum_{j=1}^{M} C_{i j} Z_{i j} \\
& \operatorname{Min} \sum_{i=1}^{N} C S_{i} \sqrt{V_{i}}+\sum_{i=1}^{N} C L_{i} \sqrt{D_{i}} \\
& D_{i}=\sum_{j=1}^{M} d_{j} z_{i j} \quad \forall i=1, \ldots, N \\
& V_{i}=\sum_{j=1}^{M} v_{j} z_{i j} \quad \forall i=1, \ldots, N \\
& \begin{array}{ll}
\sum_{j=1}^{M} d_{j} z_{i j}-I_{i}^{c a p} & \\
x_{i}, z_{i j}=\{0,1\} & \forall i=1, \ldots, N
\end{array} \\
&
\end{aligned}
$$

Equation (1) is the first objective of our biobjective problem. The idea here is to minimise both the location and the allocation cost of the resulting network. The first term is the cost of locating a specific plant/warehouse $i$, also called setup cost. The second term is associated to the cost of moving items from warehouses to customers, with $C_{i j}=T H\left(T C_{i j}+R C_{i}\right) d_{j}$ where $T H$ is the planning horizon, $T C_{i j}$ is the transport allocation cost for allocating client $j$ to warehouse $i$ and $R C_{i}$ is the transport cost associated to moving one unit of product from $i$ to $j$. The parameter $d_{j}$ is the mean demand of customer $j$.

Equation (2) is the second objective of our model. This objective aims to minimise the cost associated to the inventory management. The first term is the cost of keeping a safety stock which minimises the stock out so we can guarantee a service level at least as good as $Z_{1-\alpha}$. Here we have that $C S_{i}=T H H C Z_{1-\alpha} \sqrt{L T_{i}}$ where $H C_{i}$ corresponds to the holding cost of warehouse $i$ and $L T_{i}$ is the time between an order is placed and the products are available in the inventory. This time is also called leadtime. The variable $V_{i}$ determines the total variance of the demand for warehouse $i$. The second term in the objective function is the inventory cost, that is the cost of keeping products in stock and the administrative costs of putting an order to the suppliers, with $C L_{i}=T H \sqrt{2 H C_{i} O C_{i}}$. Here $O C_{i}$ is the order cost of warehouse $i$ and $D_{i}$ is the total demand of warehouse $i$. Constraint (5) ensures the capacity constraint of plant $i, I_{i}^{\text {cap }}$, will never be violated. As pointed out in [17], this is a very hard constraint that was relaxed in
[18]. Finally, Equation (6) states integrality (01) for the binary variables $z_{i j}$ and $x_{i}$.

\section{Multi-Objective Local Search}

Heuristic methods are a common approach to solve hard combinatorial optimisation problems such as the MO-ILM. In single objective optimisation a large number of (meta-)heuristic algorithms have been proposed in the last 50 years. This is different for MO optimisation where most of the focus has been on exact methods. Only in the last 10 years MO heuristic methods have attracted the attention of researchers.

One common approach has been extending algorithms from single objective optimisation to MO optimisation. For instance, [24] has extended simulating annealing algorithm, [13] did the same for tabu search algorithm and [2, 22] extended the steepest descent algorithm to its multi-objective version. Moreover, one field that has been very active during the last two decades is the evolutionary multi-objective optimisation (EMO). The first EMO algorithm was the vector evaluated genetic algorithm [23]. Since then several EMO approaches have been proposed in the literature $[9,11,14,27]$.

In this paper we implement the Pareto local search proposed by [2]. This method is deterministic, i.e. given an initial solution it always ends up in the same set of approximately efficient solutions of the MO problem that is being solved. The pseudo-code of the PLS algorithm is presented in Algorithm 1.

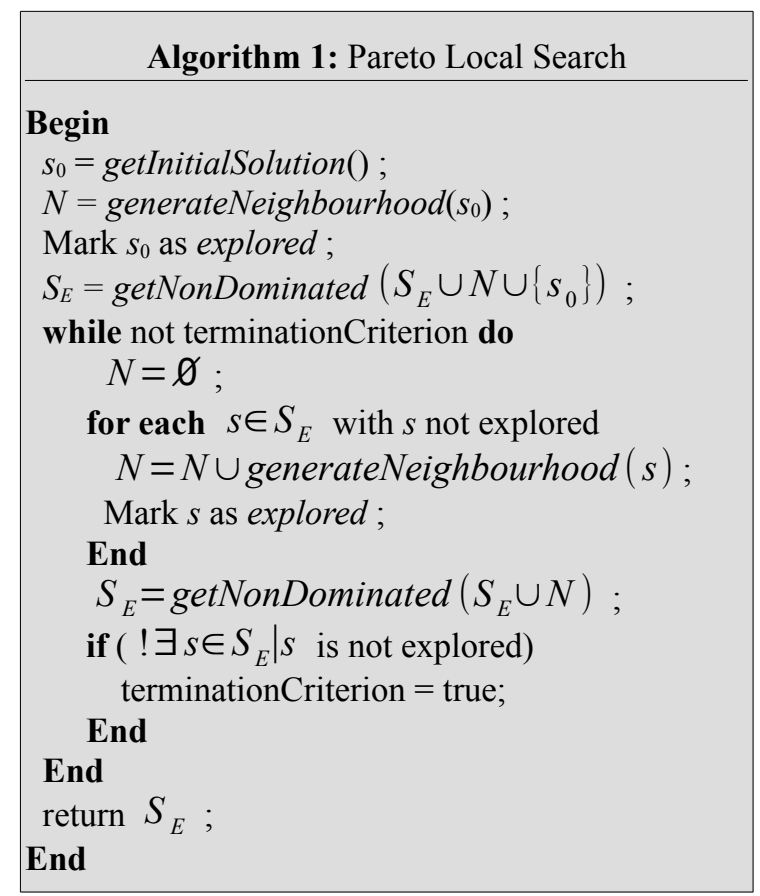


Before we explain how the implemented algorithm proceeds, we need to introduce both the neighbourhood definition and the solution representation we shall use in this paper. Let $s$ be a vector of integers where elements $s_{i}$ with $i=1, \ldots, M$ are equal to the warehouse customer $i$ is allocated to, and $M$ is the number of customers considered in the ILM problem. Thus, $s_{i}$ ranges between 1 and $N$ with $\mathrm{N}$ the number of potential warehouses. Figure 2 shows an example of a solution for a problem with 5 customers and 4 potential warehouses.

\begin{tabular}{|l|l|l|l|l|}
\hline 2 & 2 & 1 & 2 & 3 \\
\hline
\end{tabular}

Figure 2. An example of a solution representation. Here customers 1,2 and 4 are assigned to warehouse number 2. Customer 3 is assigned.

As Figure 2 shows, more than one customer can be assigned to the same warehouse while some other warehouses are left closed as no customer is assigned to them. Given this solution representation, the neighbourhood movement we consider in this paper is as follows: Solutions $s^{1}$ and $s^{2}$ are neighbours if $s_{i}^{1}=s_{i}^{2}$ for all $i$ but one. If that is the case we say that $s^{1} \in N\left(s^{2}\right)$, i.e. $s^{1}$ is within the neighbourhood of $s^{2}$. It is clear that if $s^{1} \in N\left(s^{2}\right)$, then $s^{2} \in N\left(s^{1}\right)$. Figure 3 shows two solutions $\left(s^{1}\right.$ and $\left.s^{2}\right)$ that are neighbours.

\begin{tabular}{|c|c|c|c|c|c|}
\hline$s^{1}=$ & 2 & 2 & 1 & 2 & 3 \\
\hline$s^{2}=$ & 2 & 3 & 1 & 2 & 3 \\
\hline
\end{tabular}

Figure 3. An example of two neighbour solutions. Here customer 2 has been moved from warehouse 2 to warehouse 3.

The number of elements in the neighbourhood of a solution $s$ is equal to $(N-1) \times M$. As the $N$ and $M$ terms get larger exploring the entire neighbourhood might be difficult and time consuming. For this reason we propose to set a neighbourhood size equal to 50 . Neighbours are randomly generated.

The algorithm starts by generating a feasible initial solution $s_{0} \in S$. This initial solution can be either randomly generated or provided by the decision maker. In our case we choose to generate it randomly. Once the initial solution has been generated, i.e. its objective function vector $f\left(x_{0}\right)$ has been calculated, its neighbourhood is explored as explained in the previous paragraph. After the neighbourhood of $s_{0}$ has been explored, a dominance analysis over the entire neighbourhood is performed and solution $s_{0}$ is marked as explored. Then, only those solutions that resulted in non-dominated points are kept in $S_{\mathrm{E}}$. If the set of efficient solutions $S_{\mathrm{E}}$ contains non-explored solutions, then the algorithm continue by exploring such solutions. The algorithm stops otherwise.

\section{Computational Experiments}

We present in this section the computational experiments carried out in this work. We use an Intel i5 processor and $8 \mathrm{~GB}$ of RAM to run our experiments. Linux 14.02 was the operating system. The Pareto local search algorithm was coded in JAVA 8 language using NetBeans IDE. The instance set used in this work was the same used in $[8,18]$. Since we are solving a MO problem, solutions obtained in $[8,18]$ are not necessarily efficient solutions of the MO problem. To provide a quality indicator of the obtained set of locally efficient solutions we use the hyper-volume proposed by [28]. The hyper-volume quality indicator $H$, gives the volume of the portion of the objective space that is weakly dominated by a specific set of non-dominated points $Y_{\mathrm{N}}$. Then, the higher the hyper-volume value the better the set $X_{\mathrm{E}}$ found by the algorithm.

The base instance that is presented in the results section is denoted by $B$. Just as in [18], we create four additional instances that are based on instance $B$. These additional instances are denoted by $B_{-50 \% H C}, B_{-25 \% H C}, B_{+25 \% H C}$ and $B_{+50 \% H C}$. These instances modify the holding cost of the original $B$ instance. For example, $B_{-50 \% H C}$ means holding cost $H C$ has been reduced in $50 \%$.

\subsection{Results}

A summary of the obtained results is presented in this section. Since we generate a set of solutions that are efficient for each instance, we report the number of elements in $X_{E}$ obtained by PLS (column ' $\#_{E}$ '), the number of generated solutions (column ' $\#$ ') and the hyper-volume value obtained by our algorithm (column ' $H$ '). Table 1, show the obtained results when the service level, $Z_{1-\alpha}$, is set to $97.5 \%$.

As Table 1 shows, the PLS algorithm finds a set of locally efficient vectors $x \in X_{E}$ for all the instances tested in this paper. However, only a small portion of the generated solutions resulted 
Table 1. Obtained results for $Z_{1-\alpha}=0.975$

\begin{tabular}{cccc} 
PLS & & \\
Instance & $\#_{\mathrm{E}}$ & $\#$ & $H$ \\
\hline$B_{-50 \% H C}$ & 5 & 1997 & $64.33 \%$ \\
$B_{-25 \% H C}$ & 3 & 2004 & $67.83 \%$ \\
$B$ & 8 & 1970 & $66.55 \%$ \\
$B_{+25 \% H C}$ & 7 & 1985 & $62.98 \%$ \\
$B_{+50 \% H C}$ & 7 & 1992 & $61.27 \%$ \\
\hline Average & $\mathbf{6}$ & $\mathbf{1 9 8 9 . 6}$ & $\mathbf{6 4 . 5 9 \%}$ \\
\hline
\end{tabular}

to be efficient. This might be a sign of a highly focused search, something that is known to be a drawback of MO local search algorithms. Figure 4 shows the set of generated solutions for the instance $B$. As we can see, 8 efficient solutions were generated (solid squares).

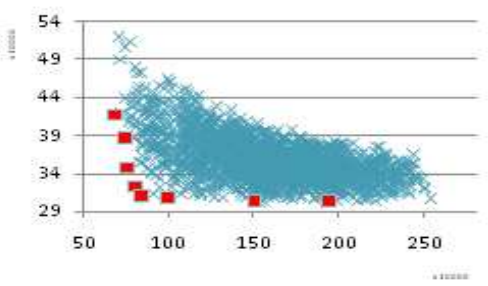

Figure 4. Generated solutions in objective space for instance $B$. Blue crosses are the generated solutions and red solid squares are the locally efficient solutions found by the PLS algorithm.

Moreover, obtained hyper-volumes are not greatly affected by changes in the holding costs. However, changing the holding cost affects the distribution of the efficient solutions in the objective space.

\section{Conclusions and Future Work}

In this article we have introduced a novel multi-objective ILM and solve it by means of a multi-objective local search called Pareto Local Search [2]. Although the bi-objective model we present here is very hard to solve, the Pareto local search algorithm implemented in this work is shown to be very effective in finding approximately efficient solutions for our problem. Other methods such as evolutionary multi-objective algorithms, multi-objective stochastic search and multi-objective swarm intelligence might be considered in order to improve solutions found by the Pareto local search implemented in this paper.

Moreover, solutions obtained for the MO-ILM suggest that there exists a trade-off between the objectives considered by the model that needs to be further studied.

\section{REFERENCES}

1. AHMADI, G., S. A. TORABI, R. TAVAKKOLI-MOGHADDAM, A Biobjective Location-inventory Model with Capacitated Transportation and Lateral Trans-shipments. Intl. J. Prod. Res., 2015, pp. 1-22.

2. ANGEL, E., E. BAMPIS, L. GOURVIES, A Dynasearch Neighborhood for the Bicriteria Traveling Salesman Problem. In X. Gandibleux, M. Sevaux, K. Sorensen, V. T'kindt, eds. Metaheuristics for Multiobjective Optimisation, vol. 535 of Lect. Notes in Ec. \& Math. Sys., Springer Verlag, 2004, pp. 153-176.

3. ARABZAD, S. M., M. GHORBANI, R., TAVAKKOLI-MOGHADDAM, An Evolutionary Algorithm for a New Multiobjective Location-inventory Model in a Distribution Network with Transportation Modes and Third-party Logistics Providers. Intl. J. Prod. Res., vol. 53(4), 2015, pp. 1038-1050.

4. ASKIN, R. G., I. BAFFO, M. XIA, Multicommodity Warehouse Location and Distribution Planning with Inventory Consideration. Intl. J. Prod. Res., vol. 52(7), 2014, pp. 1897-1910.

5. BHATTACHARYA, R., S. BANDYOPADHYAY, Solving Conflicting Bi-objective Facility Location Problem by NSGA II Evolutionary Algorithm, Intl. J. Adv. Man. Tech., vol. 51(1), 2010, pp. 397-414.

6. BRADley, J. R., B. C. ARNTZEN, The Simultaneous Planning of Production, Capacity, and Inventory in Seasonal Demand Environments. Op. Res., vol. 47(6), 1999, pp. 795-806.

7. CABRERA, G., G., P. A. MIRANDA, E. CABRERA, R. SOTO, L. J. M. RUBIO, B. CRAWFORD, F. PAREDES, Solving a Novel Inventory Location Model with Stochastic Constraints and Inventory Control Policy, Math. Prob. in Eng., vol. 2013, Article ID 670528, 12 p., 2013.

8. CABRERA, G., S. NIKLANDER, E. CABRERA, F. JOHNSON, Solving a Distribution Network Design Problem by means of Evolutionary Algorithms, SIC, vol. 25(1), 2016, pp. 21-28. 
9. COELlO COELLO, C. A., R. BECERRA, Evolutionary Multi-objective Optimization using a Cultural Algorithm. Proc. 2003 IEEE Swarm Intel. Symp., 2003, pp. 6-13.

10. DASKIN, M. S., C. R. COULLARD, Z. M. SHEN, An Inventory Location Model: Formulation, Solution Algorithm and Computational Results. Ann. Op. Res., vol. 110, 2002, pp. 83-106.

11. FONSECA, C. M., P. J. FLEMING, Genetic Algorithms for multi-objective optimization: Formulation discussion and generalization. Proc. 5th Intl. Conf. on Gen. Alg., Morgan Kaufmann Pub. Inc., 1993, pp. 416-423.

12. HAMEDANI, S., M. JABALAMELI, A. BOZORGI-AMIRI, A Location-inventory Model for Distribution Centers in a Threelevel Supply chain under Uncertainty. Intl. J. Ind. Eng. Comp., v. 4(1), 2013, pp. 93-110.

13. HANSEN, M. P. Tabu Search for Multiobjective Optimization: MOTS. In Proceedings of the Thirteenth International Conference on Multiple Criteria Decision Making, 1997, Springer-Verlag, pp. 6-10.

14. KWONLES, J., D. CORNE, The Pareto Archived Evolution Strategy: A New Baseline Algorithm for Pareto Multiobjective Optimisation. Proc. 1999 Cong. Evol. Comp., vol. 1, 1999, pp. 105.

15. LAGOS, C., F. PAREDES, S. NIKLANDER, E. CABRERA, Solving a Distribution Network Design Problem by Combining Ant Colony Systems and Lagrangian Relaxation, SIC, vol. 24 (3), 2015, pp. 251-260.

16. LIAO, S., C. HSIEH, Y. LIN, A Multiobjective Evolutionary Optimization Approach for an Integrated Locationinventory Distribution Network Problem under Vendor-managed Inventory Systems. Ann. Op. Res., v. 186(1), 2011, pp. 213-229.

17. MIRANDA, P. A., R. A. GARRIDO, Incorporating Inventory Control Decisions into a Strategic Distribution Network Design Model with Stochastic Demand. Trans. Res. Part E, pp. Log. \& Trans. Rev., vol. 40(3), 2004, pp. 183-207.

18. MIRANDA, P. A., R. A. GARRIDO, A Simultaneous Inventory Control and Facility Location Model with Stochastic
Capacity Constraints. Networks and Spatial Economics, vol. 6, 2006, pp. 39-53.

19. MIRANDA, P. A., R. A. GARRIDO, Valid Inequalities for Lagrangian Relaxation in an Inventory Location Problem with Stochastic Capacity. Trans. Res. Part E: Log. \& Trans. Rev., v. 44(1), 2008, pp. 47-65.

20. MOURITS, M., J. M. EVERS, Distribution Network Design: An Integrated Planning Support Framework. Intl. J. Phys. Dist. \& Log. Manag., vol. 25, 1995, pp. 43-57.

21. OZSEN, L., C. R. COULlARD, M. S. DASKIN, Capacitated Warehouse Location Model with Risk Pooling. Nav. Res. Log., vol. 55(4), 2008, pp. 295-312.

22. PAQUETE, L., T. SCHIAVINOTTO, T. STUTZLE, On Local Optima in Multiobjective Combinatorial Optimization Problems. Tech. Rep. AIDA-04-11, FG Intellektik, TU Darmstadt, November 2004.

23. SCHAFFER, J. D. Multiple Objective Optimization with Vector Evaluated Genetic Algorithms. Proc. 1st Intl. Conf. Gen. Alg., L. Erlbaum Assoc. Inc., 1985, pp. 93-100.

24. SERAFINI, P. Simulated Annealing for Multi- Objective Optimization Problems. Tzeng, G. H., Wang, H. F., Wen, U. P., Yu, P. L., eds. Proc. $10^{\text {th }}$ Intl. Conf. MCDM: Expand \& Enrich Domains of Thinking \& Application, 1994, Springer, pp. 283-292.

25. SHEN, Z. M., C. R. COULLARD, M. S. DASKIN, A Joint Location Inventory Model. Trans. Sc., v. 37(1), 2003, pp. 40-55.

26. SIMCHI-LEVI, D., Y. ZHAO, The Value of Information Sharing in a Two Stage Supply Chain with Production Capacity Constraints. Nav. Res. Log., vol. 50(8), 2003, pp. 888-916.

27. SRINIVAS N., K. DEB, Multi-objective Optimization using Non-dominated Sorting in Genetic Algorithms. Ev. Comp. vol. 2(3), 1994, pp. 221-248.

28. ZITZLER E., L. THIELE, Multi-objective Optimization Using Evolutionary Algorithms: A Comparative Case Study. Proc. 5th Int.l Conf. Paral. Prob. Solv. from Nature, 1998, pp. 292-304. 